\title{
General Psychiatry Does recurrent catatonia manifest in a similar fashion in all the episodes of mood disorder? A case series with literature review
}

\author{
Santanu Nath (D) , ${ }^{1}$ Rosali Bhoi, ${ }^{2}$ Biswaranjan Mishra, ${ }^{2}$ Susanta Padhy ${ }^{2}$
}

To cite: Nath S, Bhoi R,

Mishra B, et al. Does recurrent catatonia manifest in a similar fashion in all the episodes of mood disorder? A case series with literature review. General Psychiatry 2021;34:e100494. doi:10.1136/ gpsych-2021-100494

Received 29 January 2021 Accepted 16 August 2021

\section{ABSTRACT}

Catatonia, originally conceptualised by Kahlbaum in 1868, is a neuropsychiatric condition that has been found to occur concomitantly with several organic and psychiatric conditions. Starting from the era of Kraepelin and Bleuler, this condition was faultily linked with schizophrenia alone; however, over time, greater associations have been found between catatonia and mood disorders. Despite the availability of several reports supporting this finding, there is a relative paucity of studies that specifically focus on catatonia to be the first symptom manifestation heralding a subsequent mood episode. In addition, there is scant literature to determine whether there are specific presentations of catatonia that show greater associations with mood disorders and whether these signs and symptoms recur in a stereotypical fashion in the subsequent mood episodes in the lifetime of an individual. We hereby report two cases with a diagnosis of mood disorders (bipolar disorder and recurrent depressive disorder) who had catatonia as the initial symptom not only at presentation but also at subsequent episodes. The report emphasises that recurrent catatonia can be the initial clinical manifestation of an underlying mood episode, which appears otherwise masked behind the catatonic presentation. These catatonic symptoms can be interestingly similar in all the subsequent episodes. A detailed clinical evaluation is thus warranted after catatonia has been duly treated to provide a holistic management.

\section{INTRODUCTION}

Catatonia is a neuropsychiatric condition characterised by alterations in motor behaviour, thought, affect and vigilance. Originally conceptualised by Kahlbaum in 1868, catatonia was initially incorporated in dementia praecox in 1893 by Kraepelin and later in schizophrenia by Bleuler. ${ }^{1}$ Over the years, catatonia has been found to be associated with not only schizophrenia but also mood disorders and a multitude of organic brain conditions such as epilepsy, neurodegenerative disorders, tumours, haemorrhage and neuro-infections. Recent advances have led to the understanding that among psychiatric conditions, catatonia occurs more frequently with mood disorders than with non-affective psychosis such as schizophrenia. ${ }^{2}$ There are examples in the literature that point to the recurrent nature of catatonia that occurs in the setting of the relapsing-remitting course of mood disorders: the catatonia understood as a phenotypical manifestation within the particular mood episode. ${ }^{1}$ When a patient presents with recurrent catatonia, clinicians should attempt to identify the underlying conditions that otherwise remain masked under the catatonic presentation. A detailed assessment after catatonia management is always needed to diagnose the underlying psychiatric disorder, which will entail a holistic management. There is a paucity of literature that suggests catatonia to be the first phenotypical manifestation of a mood episode. We hereby report a series of two cases in which catatonia was the first symptom manifestation of all the mood episodes in both along with its persistence in a similar fashion in all the episodes. We will also briefly review the existing literature about recurrent catatonia and its association with episodes of mood disorders.

\section{CASE HISTORY}

Case 1

A 25-year-old Hindu unmarried man with unremarkable family and personal history but with past two episodes of suggestive catatonia presented with a 3-day history of remaining mute, maintaining a posture for long and refusing to interact with others along with refusal to eat and drink. His initial mental status examination (MSE) revealed catatonia (mutism, negativism and posturing) (BushFrancis Catatonia Rating Scale (BFCRS) score $=16),{ }^{3}$ and it precluded further examination of his affect, thought and perception. 
He was admitted, and on investigations, his haematological and biochemical parameters (complete haemogram; renal, hepatic and thyroid function tests; serum electrolytes; creatinine phosphokinase) and MRI of his brain were within the normal range. He was initially managed with the injection of lorazepam 4 to $8 \mathrm{mg}$ /day, which led to remission of catatonia in 3 days (BFCRS score $=0) .{ }^{3}$ On remission of catatonia, he started to show goal-directed hyperactivity and talkativeness with thought content revealing grandiosity, which gradually increased for the next few days. During this time, his MSE revealed increased speech output with prolixity, increased psychomotor activity, delusion of grandiosity and no perceptual abnormalities (Young Mania Rating Scale (YMRS) score $=26) .{ }^{4}$ Further clarification of the past two catatonia episodes revealed that in each episode, he would first present with the similar type of symptoms such as mutism, posturing and negativism (and stupor as an additional symptom in the first episode), which would be the initial presentation and have an abrupt onset with a dramatic response to treatments prescribed by a psychiatrist that would ultimately manifest in a similar mental state similar to the index episode (ie, mania). However, in none of these episodes, he continued with the psychiatrist's advice. Considering the past episodes and the current index presentation, he received a provisional diagnosis of bipolar affective disorder, current episode mania with psychosis according to ICD-10, ${ }^{5}$ and he was started on tablet sodium valproate $1 \mathrm{~g}$ /day, quetiapine $100 \mathrm{mg}$ /day and lorazepam $2 \mathrm{mg}$ /day, with which he showed significant improvement in the next 3 weeks (YMRS score $=11$ ). ${ }^{4}$ He was discharged on this regimen and on a subsequent follow-up after a month, he was found to maintain well.

\section{Case 2}

A 42-year-old Hindu married man with no significant family and personal history but with a history of catatonia thrice in the last 4 years presented with a 5-day history of remaining mute, not taking food and water, not interacting with family members and moving away from any interventions. On admission, his vital parameters were within the normal range, and his initial MSE revealed catatonia (posturing, mutism, active negativism) (BFCRS score $=18) .{ }^{3}$ His blood investigations (complete haemogram; renal, hepatic and thyroid function tests; serum electrolytes) and his MRI of the brain were within the normal range. His catatonia was not responding to lorazepam (given up to $12 \mathrm{mg}$ /day) even after the third day, and therefore, he was started on modified electroconvulsive therapy (ECT). After the second ECT, his catatonia resolved significantly (BFCRS score $=0) .{ }^{3}$ He was however found to stay alone, interacting less with people around, and on occasions, he was also seen to be tearful. A repeat MSE during this time revealed decreased speech output that was dysprosodic but relevant along with a thought content revealing delusion of sin and guilt. There were however no perceptual disturbances. Considering a severe depressive episode with psychosis (Hamilton Depression
Rating Scale (HDRS) score $=24),{ }^{6}$ he was started on olanzapine-fluoxetine $(5 \mathrm{mg}+20 \mathrm{mg}$ ) combination (OFC), and he was given a further course of six ECTs. There was significant improvement in his depressive symptoms (HDRS score $=13$ ) ${ }^{6}$ During this time, we tried to assess the causes for the previous three catatonic episodes using information shared by his wife, and it revealed that all such episodes in the last 4 years were apparently provoked with stressful life events (property dispute with relatives, financial loss, etc) after which he had similar catatonic features (mutism, negativism, posturing, withdrawal) that resolved with treatments and would be followed by low mood, lethargy, increased concern for family issues, feeling worthless, anorexia and hypersomnolence. His provisional diagnosis was hence changed to recurrent depressive disorder with current episode of severe depression with catatonia. He was discharged on OFC, and on a follow-up after 2 weeks, and a month later he was found to be well. We however got a call from his wife 4 months later that there was another similar episode for which he was taken to a tertiary care psychiatric hospital and was managed in similar lines. We however planned to follow him prospectively having a suspicion whether he may land up in a bipolar disorder diagnosis in future.

\section{DISCUSSION}

Catatonia has long been thought to be associated more with schizophrenia, starting from the era of Kraepelin and Bleuler. However, Kahlbaum, who initially gave the descriptions of catatonia along with its types (table 1) in 1868, conceptualised it to be associated mostly with mood disorders. ${ }^{1}$ Over the years, its association with mood disorders has been strengthened. Catatonic signs have consistently been reported with mood disorders in $13 \%$ to $31 \%$ cases. $^{7}$ Various systematic studies reported that, among hospitalised manic patients, more than $25 \%$ meet the criteria for catatonia, whereas among patients with catatonia, more than half meet the criteria for a manic-depressive illness. ${ }^{8}$ The seminal paper of Barnes et at also favoured this association. Thirty-six per cent of 25 patients with catatonia whom they described over a span of 12 years had affective disorders, whereas only $4 \%$ had a diagnosis of schizophrenia. The remaining $(60 \%)$ had either an organic brain

\begin{tabular}{ll}
\hline $\begin{array}{l}\text { Table 1 } \\
\text { Catatonia } \\
\text { types }\end{array}$ & Clinical descriptions \\
\hline $\begin{array}{l}\text { Catatonia } \\
\text { mitis }\end{array}$ & $\begin{array}{l}\text { Mild form, rapid development, associated } \\
\text { with depression, good prognosis. }\end{array}$ \\
$\begin{array}{l}\text { Catatonia } \\
\text { gravis }\end{array}$ & $\begin{array}{l}\text { Severe form, relatively rapid development, } \\
\text { associated with pure mania and mixed } \\
\text { states, good prognosis. }\end{array}$ \\
$\begin{array}{l}\text { Catatonia } \\
\text { protracta }\end{array}$ & $\begin{array}{l}\text { Severe form, insidious onset, bizarre } \\
\text { movements and behaviours, chronic, poor } \\
\text { prognosis. }\end{array}$ \\
\hline
\end{tabular}


condition or no concomitant diagnosis. The affective disorders were all recurrent depressive disorders having mostly recurrent catatonia in the episodes. What was also striking was the fact that such a presentation (recurrent catatonia with depression) was also present in their family members, which points to its high genetic loading. Usman $e t a l^{10} \mathrm{did}$ a retrospective chart review of 13968 patients who presented with catatonia in their tertiary care neuropsychiatry hospital in Nigeria and found an increase in the diagnosis of mood disorders (from $10 \%$ to $20.7 \%$ ) in a span of 20 years alongside a decrement of a schizophrenia diagnosis (from $82.5 \%$ to $53.4 \%$ ) during the same period. The patients with mood disorders were mostly females, but there was no significant difference in the sociodemographic profile in these two diagnostic groups. Parker et al also held the same opinion in their review, reporting most inpatients with catatonia having a comorbid mood disorder ( $36 \%$ to $63.2 \%$ ) than a psychotic disorder (29.8\% to $32 \%) .{ }^{11}$ Within bipolar disorder, catatonia has been reported to be more associated with mixed states in comparison with a manic episode as reported in two studies that found $61 \%$ and $73 \%$ of patients with catatonia have an index mixed episode, respectively. ${ }^{13}$ Catatonia has also been reported with atypical presentations of bipolar disorders. Mahmoudi $e t a l^{14}$ reported a case of an older man with 'atypical bipolar disorder' who had a family history of mood disorders and who presented with recurrent hypersomnia, autonomic instability along with stupor, mutism, negativism and agitation during such episodes. He received a diagnosis of catatonia and atypical bipolar disorder and responded significantly with mood stabiliser use. Kruger and Bräunig proposed some commonalities in the descriptions of catatonia in mood disorders, especially mania. According to them, catatonia is mostly associated with mixed states, they are transient and are markers of severity of mood disorders but still have a better prognosis than catatonic schizophrenia. ${ }^{15}$

The two cases discussed above had mood disorders (bipolar effective disorder in case 1 and recurrent depressive disorder in case 2), and for both of them, the initial symptoms were those of catatonia, the treatment of which ultimately led to the emergence of the underlying mood symptoms. What was also strikingly noticeable was the recurrence of catatonia in a similar fashion (their initial most presentation and the same catatonic signs and symptoms) in all their subsequent mood episodes. Some important observations were pointed out: (1) catatonia can mask a mood episode, which is consistent in subsequent episodes whether it is a bipolar illness or a recurrent depressive disorder; and (2) the similarity in its pattern and typology in the later episodes, for example, mutism, negativism and posturing in our cases. The current report thus points to the need for looking beyond such type of catatonic presentations, more so when they are the first presenting symptoms and occurring recurrently in future episodes. A thorough assessment after treating the initial catatonia can unmask an affective disorder that warrants a holistic management. Usman et $a l$ in their retrospective chart review reported that among many catatonic symptoms, mutism $(87.5 \%)$, staring (50\%), withdrawal (43.8\%) and posturing/catalepsy (56.3\%) were the most frequent and most consistent symptoms in patients with mood disorder when compared with schizophrenia. ${ }^{10}$ Francis et al, however, reported a consistency of motor symptoms across all the catatonic episodes in the five patients they reported, although only one received a diagnosis of mood disorder among them. ${ }^{16}$ There are pieces of literature evidence to support that the 'retarded' type of catatonia is highly associated with mood disorders. Our cases as described above both had retarded type of catatonia with mutism, negativism, posturing and withdrawal being their manifested signs. Peralta et al endeavoured to dissect the catatonic phenotype in psychosis and mood disorders and came to the conclusion that 'psychomotor retardation' was the most frequent catatonic sign in their cohort followed by rigidity and mutism, which had the highest familiarity. ${ }^{17}$ Lin et $a l,{ }^{18}$ in their 15 -year retrospective record review of 30 patients with a history of recurrent catatonia, found that $6.7 \%$ and $16.7 \%$ of patients had a diagnosis of bipolar disorder and depressive disorder, respectively. All had 'retarded' type of catatonic symptoms and signs viz stupor and mutism, the Kahlbaum syndrome. The higher prevalence of mutism, stupor, negativism and withdrawal was also reported in a recent study on a naturalistic setup involving 26 patients with catatonia having bipolar disorder. ${ }^{12}$ All these evidences point to a possible association of retarded catatonia with mood disorders and a consistency of their manifestation across future mood episodes (whether manic or depressive). However, further research is warranted that will longitudinally follow up patients with mood disorders, who had catatonic presentation during the episodes, to systematically look into the consistency of the catatonic signs and symptoms in their lifetime mood episodes.

\section{Twitter Santanu Nath @doc_santanu}

Contributors SN, BM and SP assessed the patients described in the manuscript and managed them. RB and SN wrote the initial manuscript draft, which was revised by BM and SP. The final manuscript was seen and reviewed by all the authors.

Funding The authors have not declared a specific grant for this research from any funding agency in the public, commercial or not-for-profit sectors.

Competing interests None declared.

Patient consent for publication Not required.

Provenance and peer review Not commissioned; externally peer reviewed.

Open access This is an open access article distributed in accordance with the Creative Commons Attribution Non Commercial (CC BY-NC 4.0) license, which permits others to distribute, remix, adapt, build upon this work non-commercially, and license their derivative works on different terms, provided the original work is properly cited, appropriate credit is given, any changes made indicated, and the use is non-commercial. See: http://creativecommons.org/licenses/by-nc/4.0/.

ORCID iD

Santanu Nath http://orcid.org/0000-0001-5357-3783

\section{REFERENCES}

1 Fink M. Catatonia: a syndrome appears, disappears, and is rediscovered. Can J Psychiatry 2009;54:437-45.

2 Rajagopal S. Catatonia. Adv Psychiatr Treat 2007;13:51-9.

3 Bush G, Fink M, Petrides G, et al. Catatonia. I. rating scale and standardized examination. Acta Psychiatr Scand 1996;93:129-36. 
4 Young RC, Biggs JT, Ziegler VE, et al. A rating scale for mania: reliability, validity and sensitivity. Br J Psychiatry 1978;133:429-35.

5 The ICD-10. classification of mental and behavioural disorders clinical descriptions and diagnostic guidelines ; ICD-10. Geneva: WHO, 1992.

6 Hamilton M. A rating scale for depression. J Neurol Neurosurg Psychiatry 1960;23:56-62.

7 Caroff S, Mann S, Francis S. Catatonia: from psychopathology to neurobiology. Washington, DC, London, England: American Psychiatric Publishing, 2004.

8 Fink M, Taylor MA. Catatonia: a clinician's guide to diagnosis and treatment. New York City, NY: Cambridge University Press, 2003.

9 Barnes MP, Saunders M, Walls TJ, et al. The syndrome of Karl Ludwig Kahlbaum. J Neurol Neurosurg Psychiatry 1986;49:991-6.

10 Usman DM, Olubunmi OA, Taiwo O, et al. Comparison of catatonia presentation in patients with schizophrenia and mood disorders in Lagos, Nigeria. Iran J Psychiatry 2011;6:7-11.

11 Parker G, McClure G, Paterson A. Melancholia and catatonia: disorders or specifiers? Curr Psychiatry Rep 2015;17:536.
12 Medda P, Toni C, Luchini F, et al. Catatonia in 26 patients with bipolar disorder: clinical features and response to electroconvulsive therapy. Bipolar Disord 2015;17:892-901.

13 Krüger S, Cooke RG, Spegg CC, et al. Relevance of the catatonic syndrome to the mixed manic episode. J Affect Disord 2003;74:279-85.

14 Mahmoudi M, Friedman D, Vendrame M, et al. A case of recurrent hypersomnia with autonomic dysfunction. J Clin Sleep Med 2017;13:923-4.

15 Krüger S, Bräunig P. Catatonia in affective disorder: new findings and a review of the literature. CNS Spectr 2000;5:48-53.

16 Francis A, Divadeenam KM, Bush G, et al. Consistency of symptoms in recurrent catatonia. Compr Psychiatry 1997;38:56-60.

17 Peralta V, Fañanás L, Martín-Reyes M, et al. Dissecting the catatonia phenotype in psychotic and mood disorders on the basis of familialgenetic factors. Schizophr Res 2018;200:20-5.

18 Lin C-C, Hung Y-Y, Tsai M-C, et al. Relapses and recurrences of catatonia: 30-case analysis and literature review. Compr Psychiatry 2016;66:157-65.

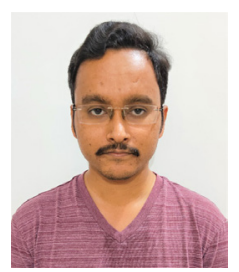

Dr. Santanu Nath completed his graduation in Medicine (MBBS) from Calcutta National Medical College, Kolkata, West Bengal, India, in 2011. He completed his post-graduation in Psychiatry (MD) from Lokopriya Gopinath Bordoloi (LGB) Regional Institute of Mental Health (LGBRIMH), Tezpur, Assam, India, in 2016 and then went on to earn his Diplomate of National Board (DNB) degree in Psychiatry from the National Board of Examinations (NBE), New Delhi, India, in 2019. After attaining his MD degree, he did his Senior Residency in Psychiatry in All India Institute of Medical Sciences (AIIMS), Bhubaneswar, Odisha, India, and then he worked as an Assistant Professor at the Department of Psychiatry in AIIMS, Bhubaneswar, until February 2021. He has been working as an Assistant Professor at the Department of Psychiatry in Institute of Medical Sciences, Banaras Hindu University (BHU), Varanasi, India, since February 2021. He is a Life Ordinary Member of the Indian Psychiatric Society and a member of the International Headache Society. He has publications in both national and international psychiatric journals, which comprise randomized clinical trials and commentaries. He is also the reviewer of two national and international indexed psychiatry journals. His main research interests include psychopharmacology, obsessive-compulsive disorder, treatment resistance in psychiatry and organic psychiatry. 